Journal of Algebraic Combinatorics, 18, 189-209, 2003 2004 Kluwer Academic Publishers. Manufactured in The Netherlands.

\title{
Some Identities for Enumerators of Circulant Graphs
}

\author{
VALERY LISKOVETS \\ Institute of Mathematics, National Academy of Sciences, 220072, Minsk, Belarus
}

liskov@im.bas-net.by

Received August 16, 2001; Revised January 8, 2003; Accepted January 21, 2003

\begin{abstract}
We establish analytically several new identities connecting enumerators of different types of circulant graphs mainly of prime, twice prime and prime-squared orders. In particular, it is shown that the half-sum of the number of undirected circulants and the number of undirected self-complementary circulants of prime order is equal to the number of directed self-complementary circulants of the same order. Several identities hold only for prime orders $p$ such that $(p+1) / 2$ is also prime. Some conjectured generalizations and interpretations are discussed.
\end{abstract}

Keywords: cycle index, cyclic group, nearly doubled primes, Cunningham chain, self-complementary graph, regular tournament

Mathematics Subject Classification (2000): $\quad$ 05C30, 05A19, 11A41

\section{Introduction}

1.1. Motivation. Identities considered in this paper connect different enumerators of circulant graphs mainly of prime, twice prime and prime-squared orders. The idea of this paper goes back to the article [10], where we counted uniformly circulants of five kinds and derived several identities. Here we consider six types of circulants: directed, undirected and oriented circulants (specified by valency or not), and self-complementary circulants of the same types. Most of the obtained identities may be called analytical (or formal) in the sense that they rest exclusively on the enumerative formulae and follow from special properties of the cycle indices of regular cyclic groups. It is more difficult to discover such an identity than to prove it analytically. Almost all of the identities were first revealed and conjectured based on numerical observations.

From the combinatorial point of view, the identities look rather strange. They are very simple but no structural or algebraic properties of circulants are used to derive them (with few exceptions), nor do we establish bijective proofs. The latter task is challenging although in some cases the existence of a natural bijection between participating circulants seems unlikely. Of course there may exist other combinatorial or algebraic explanations or interpretations of the identities.

Several identities hold only for a special type of prime orders $p$, namely, those for which $\frac{p+1}{2}$ is also prime. Such primes are familiar in number theory. Probably this is the first combinatorial context where they play a substantial role. 
We cover here numerous identities that have been obtained previously and deduce about ten new ones. We deliberately represent new identities in different equivalent forms and formulate simple corollaries keeping in mind possible future generalizations and combinatorial proofs. Some of the derived identities look more elegant than the original ones.

The present paper is partially based upon the work [11] that contains detailed enumerative formulae for circulants, extensive tables and several identities. We reproduce all necessary results from it, and our exposition is basically self-contained.

1.2. Definitions. Let $n$ be a positive integer, $\mathbb{Z}_{n}:=\{0,1,2, \ldots, n-1\}$. We denote by $\mathbb{Z}_{n}^{*}$ the set of numbers in $\mathbb{Z}_{n}$ relatively prime to $n$ (that is invertible elements modulo $n$ ). So, $\left|\mathbb{Z}_{n}^{*}\right|=\phi(n)$, where $\phi(n)$ is the Euler totient function. $Z(n)$ denotes a regular cyclic permutation group of order and degree $n$, i.e., the group generated by an $n$-cycle.

The cycle index of $Z(n)$ is the polynomial

$$
\mathcal{J}_{n}(\mathbf{x})=\frac{1}{n} \sum_{r \mid n} \phi(r) x_{r}^{n / r},
$$

where $\mathbf{x}$ stands for the sequence of variables $x_{1}, x_{2}, x_{3}, \ldots$

The term "graphs" means both undirected and directed graphs. We consider only simple graphs, that is graphs without loops, multiple edges, or multiple arcs. An $n$-graph means a graph of order $n$, where the order means the number of vertices. We refer to Harary [9] for terminology concerning graphs.

An (undirected) edge is identified with the pair of the corresponding oppositely directed arcs. Accordingly, an undirected graph is considered to be a (symmetric) digraph. On the contrary, a digraph is oriented if it has no pair of oppositely directed arcs.

A circulant graph of order $n$, or simply a circulant, means a graph $\Gamma$ on the vertex set $\mathbb{Z}_{n}$ which is invariant with respect to the cyclic permutation $(0,1,2, \ldots, n-1)$, i.e., if $(u, v)$ is an edge of $\Gamma$ then so is $(u+1, v+1)$. In other words, this is a Cayley graph with respect to the cyclic group $\mathbb{Z}_{n}$. Every circulant is a regular graph of some valency $r$.

A circulant $\Gamma$ is specified by the set $X=X(\Gamma)$ (called its connection set) of all vertices adjacent to the vertex 0 . $\Gamma$ is undirected iff $X$ is symmetric, which means that $-X=X$, where $-X:=\{-v \mid v \in X\}$. On the contrary, $\Gamma$ is oriented iff $X$ is anti-symmetric, that is, $X \cap(-X)=\emptyset$. Such a circulant is a tournament iff $X$ is complete, that is, $X \cup(-X)=\mathbb{Z}_{n}^{\prime}$, where $\mathbb{Z}_{n}^{\prime}:=\mathbb{Z}_{n} \backslash\{0\}$. The complement of $\Gamma$ is the circulant $\Gamma^{\prime}$ with the connection set $X^{\prime}:=\mathbb{Z}_{n}^{\prime} \backslash X$.

Regular self-complementary graphs are of valency $r=(n-1) / 2$ and, thus, exist only for odd $n$. Moreover, an undirected self-complementary $n$-circulant can exist only if $4 \mid(n-1)$ since it contains $n(n-1) / 4$ edges. It is easy to see that any circulant tournament is selfcomplementary.

Graphs are considered here up to isomorphism. We deal with the enumerators of (nonisomorphic) circulants of several types. For convenience, the type is written as the subscript. Henceforth:

- $C_{\mathrm{d}}(n)$ denotes the number of directed circulant graphs;

- $C_{\mathrm{u}}(n)$ denotes the number of undirected circulant graphs; 
- $C_{\mathrm{o}}(n)$ denotes the number of oriented circulant graphs;

- $C_{\mathrm{sd}}(n)$ and $C_{\mathrm{su}}(n)$ denote the numbers of self-complementary directed and undirected circulant graphs respectively;

- $C_{\mathrm{t}}(n)$ denotes the number of circulant tournaments;

- $C_{\mathrm{d}}(n, r), C_{\mathrm{u}}(n, r)$ and $C_{\mathrm{o}}(n, r)$ denote the corresponding numbers of circulants of order $n$ and valency $r$ while $c_{\mathrm{d}}(n, z), c_{\mathrm{u}}(n, z)$ and $c_{\mathrm{o}}(n, r)$ are their generating functions by valency (polynomials in $z$ ):

$$
\begin{aligned}
& c_{\mathrm{d}}(n, z):=\sum_{r \geq 0} C_{\mathrm{d}}(n, r) z^{r}, \quad c_{\mathrm{u}}(n, z):=\sum_{r \geq 0} C_{\mathrm{u}}(n, r) z^{r}, \\
& c_{\mathrm{o}}(n, z):=\sum_{r \geq 0} C_{\mathrm{o}}(n, r) z^{r} .
\end{aligned}
$$

Clearly

$$
C_{\mathrm{d}}(n)=c_{\mathrm{d}}(n, 1), \quad C_{\mathrm{u}}(n)=c_{\mathrm{u}}(n, 1) \quad \text { and } \quad C_{\mathrm{o}}(n)=c_{\mathrm{o}}(n, 1) .
$$

These quantities and the corresponding circulants are considered in more detail in $[10,11]$. In particular, the following simple uniform enumerative formulae have been obtained there:

1.3 Theorem (counting circulants of prime and twice prime order) For $p$ an odd prime,

$$
\begin{aligned}
& \left.c_{\mathrm{d}}(p, z)=\left.\mathcal{J}_{p-1}(\mathbf{x})\right|_{\left\{x_{r}:=1+z^{r}\right.}\right\}_{r=1,2, \ldots} \\
& c_{\mathrm{u}}(p, z)=\left.\mathcal{J}_{\frac{p-1}{2}}(\mathbf{x})\right|_{\left\{x_{r}:=1+z^{2 r}\right\}_{r=1,2, \ldots}} \\
& c_{\mathrm{o}}(p, z)=\left.\mathcal{J}_{p-1}(\mathbf{x})\right|_{\left\{x_{r}:=1\right\}_{r \text { even }},\left\{x_{r}^{2}:=1+2 z^{r}\right\}_{\text {odd }}} \\
& C_{\text {sd }}(p)=\left.\mathcal{J}_{p-1}(\mathbf{x})\right|_{\left\{x_{r}:=2\right\}_{r_{\text {even }}},\left\{x_{r}:=0\right\}_{r \text { odd }}} \\
& C_{\mathrm{su}}(p)=\left.\mathcal{J}_{\frac{p-1}{2}}(\mathbf{x})\right|_{\left\{x_{r}:=2\right\}_{r} \text { even }},\left\{x_{r}:=0\right\}_{r} \text { odd } \\
& C_{\mathrm{t}}(p)=\left.\mathcal{J}_{p-1}(\mathbf{x})\right|_{\left\{x_{r}:=0\right\}_{r \text { even }},\left\{x_{r}^{2}:=2\right\}_{r} \text { odd }} \\
& c_{\mathrm{d}}(2 p, z)=\left.\mathcal{J}_{p-1}(\mathbf{x})\right|_{\left\{x_{r}:=\left(1+z^{r}\right)^{2}\right\}_{r=1,2, \ldots}} \cdot(1+z) \\
& c_{\mathrm{u}}(2 p, z)=\left.\mathcal{J}_{\frac{p-1}{2}}(\mathbf{x})\right|_{\left\{x_{r}:=\left(1+z^{2 r}\right)^{2}\right\}_{r=1,2, \ldots}} \cdot(1+z) \\
& c_{\mathrm{o}}(2 p, z)=\left.\mathcal{J}_{p-1}(\mathbf{x})\right|_{\left\{x_{r}:=1\right\}_{r \text { even }},\left\{x_{r}:=1+2 z^{r}\right\}_{r \text { odd }}} .
\end{aligned}
$$

\section{Cycle indices of cyclic groups}

2.1. There are several technical formulae connecting the cycle indices $\mathcal{J}_{\frac{p-1}{2}}$ and $\mathcal{J}_{p-1}$. They are interesting per se and will be used in the proofs of subsequent identities.

For any natural $m$, we set

$$
m:=2^{k} m^{\prime}
$$

where $m^{\prime}$ is odd. 
In the polynomial $\mathcal{J}_{2 m}$ we first distinguish the terms corresponding to the divisors $r$ with the highest possible power of 2, i.e., $k+1$ :

$$
\mathcal{J}_{2 m}(\mathbf{x})=\frac{1}{2 m} \sum_{r \mid 2 m} \phi(r) x_{r}^{2 m / r}=\frac{1}{2 m}\left(\sum_{r \mid m} \phi(r) x_{r}^{2 m / r}+\sum_{r \mid m^{\prime}} \phi\left(2^{k+1} r\right) x_{2^{k+1} r}^{m^{\prime} / r}\right) .
$$

After easy transformations taking into account that $\phi\left(2^{k+1} r\right)=2^{k} \phi(r)$ for odd $r$ and $k \geq 0$ we obtain

\subsection{Lemma}

$$
2 \mathcal{J}_{2 m}(\mathbf{x})=\mathcal{J}_{m}\left(\mathbf{x}^{2}\right)+\mathcal{J}_{m^{\prime}}\left(\mathbf{x}_{(k+1)}\right)
$$

where $\mathbf{x}^{2}:=x_{1}^{2}, x_{2}^{2}, x_{3}^{2}, \ldots$ and $\mathbf{x}_{(k+1)}:=x_{2^{k+1}}, x_{2 \cdot 2^{k+1}}, x_{3 \cdot 2^{k+1}} \ldots$

Now in $\mathcal{J}_{m}(\mathbf{x})$ we partition the set of divisors with respect to powers of 2:

$$
\mathcal{J}_{m}(\mathbf{x})=\frac{1}{m}\left(\sum_{r \mid m^{\prime}} \phi(r) x_{r}^{2^{k} m^{\prime} / r}+\sum_{i=1}^{k} \sum_{r \mid m^{\prime}} 2^{i-1} \phi(r) x_{2^{i} r}^{2^{k-i} m^{\prime} / r}\right)
$$

and we do the same for $\mathcal{J}_{2 m}(\mathbf{x})$. Comparing similar terms in both formulae, we easily arrive at the following:

$$
\mathcal{J}_{m}(\mathbf{x})=\mathcal{J}_{2 m}\left(0, x_{1}, 0, x_{2}, 0, x_{3}, 0, \ldots\right)+\frac{1}{2 m} \sum_{r \mid m^{\prime}} \phi(r) x_{r}^{m / r}
$$

The second summand on the right-hand side of formula (2.2) can be represented in different useful forms. First of all, it is evidently equal to $\frac{1}{2 m} \sum_{\substack{r \mid m \\ r \text { odd }}} \phi(r) x_{r}^{m / r}$ and also to $\frac{1}{2} \mathcal{J}_{m}\left(x_{1}, 0, x_{3}, 0, x_{5}, 0, \ldots\right)$. Hence

$$
\mathcal{J}_{m}(\mathbf{x})=\mathcal{J}_{2 m}\left(0, x_{1}, 0, x_{2}, 0, x_{3}, 0, \ldots\right)+\frac{1}{2} \mathcal{J}_{m}\left(x_{1}, 0, x_{3}, 0, x_{5}, 0, \ldots\right)
$$

Every term in $\mathcal{J}_{m}$ contains only one variable. Therefore

$$
\mathcal{J}_{m}\left(x_{1}, 0, x_{3}, 0, x_{5}, 0, \ldots\right)=\mathcal{J}_{m}(\mathbf{x})-\mathcal{J}_{m}\left(0, x_{2}, 0, x_{4}, 0, x_{6}, 0, \ldots\right) .
$$

Hence by (2.3) we have

\subsection{Lemma}

$$
2 \mathcal{J}_{2 m}\left(0, x_{1}, 0, x_{2}, 0, x_{3}, 0, \ldots\right)=\mathcal{J}_{m}(\mathbf{x})+\mathcal{J}_{m}\left(0, x_{2}, 0, x_{4}, 0, x_{6}, 0, \ldots\right),
$$


that is,

$$
\left.2 \mathcal{J}_{2 m}(\mathbf{y})\right|_{\left\{y_{r}:=0\right\}_{r \text { odd }},\left\{y_{r}:=x_{r / 2}\right\}_{r \text { even }}}=\mathcal{J}_{m}(\mathbf{x})+\left.\mathcal{J}_{m}(\mathbf{y})\right|_{\left\{y_{r}:=0\right\}_{r \text { odd }},\left\{y_{r}:=x_{r}\right\}_{r \text { even }}} .
$$

Now $\mathcal{J}_{m}\left(x_{1}, 0, x_{3}, 0, x_{5}, 0, \ldots\right)=2 \mathcal{J}_{2 m}\left(\sqrt{x_{1}}, 0, \sqrt{x_{3}}, 0, \sqrt{x_{5}}, 0, \ldots\right)$. Therefore by $(2.3)$,

$$
\mathcal{J}_{m}(\mathbf{x})=\mathcal{J}_{2 m}\left(0, x_{1}, 0, x_{2}, 0, x_{3}, 0, \ldots\right)+\mathcal{J}_{2 m}\left(\sqrt{x_{1}}, 0, \sqrt{x_{3}}, 0, \sqrt{x_{5}}, 0, \ldots\right) .
$$

Since the non-zero variables in both right-hand side summands alternate, one may join them into a single cycle index. This transformation gives rise to the following expression:

\subsection{Lemma}

$$
\mathcal{J}_{m}(\mathbf{x})=\mathcal{J}_{2 m}\left(\sqrt{x_{1}}, x_{1}, \sqrt{x_{3}}, x_{2}, \sqrt{x_{5}}, x_{3}, \ldots\right) .
$$

In other words, $\mathcal{J}_{m}(\mathbf{x})=\left.\mathcal{J}_{2 m}(\mathbf{y})\right|_{\left\{y_{r}^{2}:=x_{r}\right\}_{r \text { odd }},\left\{y_{r}:=x_{r / 2}\right\}_{r \text { even }}}$.

Finally we need one further formula. Substituting (2.5) into (2.4) we obtain

$$
\begin{aligned}
\mathcal{J}_{2 m}\left(0, x_{1}, 0, x_{2}, 0, x_{3}, \ldots\right)= & \mathcal{J}_{2 m}\left(\sqrt{x_{1}}, 0, \sqrt{x_{3}}, 0, \sqrt{x_{5}}, \ldots\right) \\
& +\mathcal{J}_{m}\left(0, x_{2}, 0, x_{4}, 0, x_{6}, \ldots\right) .
\end{aligned}
$$

\section{Known identities}

3.1. Let $p>3$ be a prime such that $q=\frac{p+1}{2}$ is also prime. Then by Klin-LiskovetsPöschel [10],

$$
c_{\mathrm{u}}(p, z)=c_{\mathrm{d}}\left(\frac{p+1}{2}, z^{2}\right),
$$

that is, $C_{\mathrm{u}}(p, 2 r)=C_{\mathrm{d}}\left(\frac{p+1}{2}, r\right), r \geq 0$, and

$$
C_{\mathrm{su}}(p)=C_{\mathrm{sd}}\left(\frac{p+1}{2}\right)
$$

These equalities follow directly from Theorem 1.3 and are in fact the first formal (i.e., analytically proved) identities for enumerators of circulants.

We note that

$$
p-1=2(q-1)
$$

which explains the particular role of such primes in our considerations. 
It follows from (3.1) that

$$
C_{\mathrm{u}}(p)=C_{\mathrm{d}}\left(\frac{p+1}{2}\right)
$$

As a matter of fact, these identities are valid for $p=3$ as well.

3.2. If $p>3$ is a prime such that $q=\frac{p+1}{2}$ is also prime, then

$$
2 c_{\mathrm{o}}(p, z)=c_{\mathrm{o}}(p+1, z)+1 \text {. }
$$

Proof (cf. [11]): Identity (3.3) follows directly from Theorem 1.3 (the third and ninth formulae) and from the polynomial equality

$$
2 \mathcal{J}_{2 m}^{\prime}(\mathbf{x})=\mathcal{J}_{m}^{\prime}\left(\mathbf{x}^{2}\right)+1
$$

for an arbitrary $m$ where $\mathcal{J}_{m}^{\prime}(\mathbf{x}):=\left.\mathcal{J}_{m}(\mathbf{x})\right|_{\left\{x_{r}:=1\right\}_{\text {reven }}}$. This equality is a particular case of expression $(2.1)$ since $\mathcal{J}_{m}(1,1,1, \ldots)=1$. Here we put $2 m:=p-1$ (hence $m=q-1$ ).

Putting $z:=1$ in (3.3) we obtain

$$
2 C_{\mathrm{o}}(p)=C_{\mathrm{o}}(p+1)+1
$$

3.3. According to [10],

$$
C_{\mathrm{su}}(n)=0
$$

and

$$
C_{\mathrm{sd}}(n)=C_{\mathrm{t}}(n)
$$

if $n=p$ or $p^{2}$ and $p \equiv 3(\bmod 4)$.

Next, combining (3.5) with (3.2) we obtain

$$
C_{\mathrm{su}}(p)=C_{\mathrm{t}}\left(\frac{p+1}{2}\right)
$$

if both $p$ and $\frac{p+1}{2}$ are primes and $p \equiv 5(\bmod 8)$.

3.4. For any prime $p$,

$$
C_{\mathrm{sd}}(p)=C_{\mathrm{t}}(p)+C_{\mathrm{su}}(p) .
$$


Since tournaments and undirected self-complementary circulants are particular cases of directed self-complementary circulants (hence in general $C_{\mathrm{sd}}(n) \geq C_{\mathrm{t}}(n)+C_{\mathrm{su}}(n)$ ), equality (3.7) has a simple interpretation: any directed self-complementary circulant graph of prime order is either anti-symmetric (a tournament) or symmetric (an undirected graph). This beautiful claim was first established by Chia-Lim [4] by means of simple algebraic arguments. But in view of Theorem 1.3 (the fourth, fifth and sixth formulae), identity (3.7) for odd $p$ is a direct consequence of formula (2.7): merely substitute 2 for all variables $x_{1}, x_{2}, x_{3}, \ldots$ (and (3.7) is trivial for $p=2$ ).

3.5. According to Fronček-Rosa-Šiŕaň [8] (see also [1]), undirected self-complementary circulants of order $n$ exist if and only if all prime divisors $p$ of $n$ are congruent to 1 modulo 4 . Hence (3.4) holds if there is a prime $p \mid n, p \equiv 3(\bmod 4)$.

3.6. For composite orders, directed self-complementary circulants that are neither tournaments nor undirected graphs do exist but are comparatively rare. They are called mixed. The least suitable order is $15: C_{\mathrm{sd}}^{\text {mixed }}(15):=C_{\mathrm{sd}}(15)-C_{\mathrm{su}}(15)-C_{\mathrm{t}}(15)=20-0-16=4$ (see Table 1 in the Appendix). We will return to mixed circulants in Sections 5 and 7.

3.7. The last known non-trivial identity concerns undirected circulants of even order and odd valency:

$$
C_{\mathrm{u}}(2 n, 2 r+1)=C_{\mathrm{u}}(2 n, 2 r)
$$

for any $n$ and $r$. This identity is known to hold for square-free $n$. Moreover it has been verified for all orders less 54 and is conjectured to be valid for all even orders [16]. We will return to this conjecture in the last section.

3.8. There are also two useful but trivial valency-dependent identities:

$$
C_{\mathrm{u}}(2 n+1,2 r+1)=0,
$$

which is valid since an undirected graph of odd order cannot have all vertices of odd valency, and

$$
C_{\mathrm{i}}(n, n-r-1)=C_{\mathrm{i}}(n, r), \quad \mathrm{i}=\mathrm{u} \text { or } \mathrm{d},
$$

which is valid by graph complementation.

\section{New identities for circulants of prime order}

4.1 Proposition For prime $p$,

$$
2 C_{\mathrm{sd}}(p)=C_{\mathrm{u}}(p)+C_{\mathrm{su}}(p) .
$$

In particular,

$$
C_{\mathrm{u}}(p)=2 C_{\mathrm{sd}}(p)=2 C_{\mathrm{t}}(p) \quad \text { if } p \equiv 3 \quad(\bmod 4) .
$$


Proof: For $p>2$, substitute 2 for all variables in formula (2.4) with $p-1=2 m$. By Theorem 1.3 (the fourth, second and fifth formulae) and formula (1.2), we immediately obtain (4.1). Clearly the second summand in (2.4) vanishes if $m$ is odd (see (3.4)).

In Section 6.2 we will obtain a generalization of $(4.1 \mathrm{a})$ to $p \equiv 1(\bmod 4)$.

\subsection{Remarks}

1. Despite the fact that all participating quantities (and the corresponding numerical values for small $p$ ) have been known long ago, this striking identity has evidently escaped attention of the previous researchers including the present author. I do not know whether it can be generalized to non-prime orders.

2. In view of equation (3.7), identity (4.1) can be represented equivalently in the following form:

$$
C_{\mathrm{u}}(p)=C_{\mathrm{sd}}(p)+C_{\mathrm{t}}(p)=C_{\mathrm{su}}(p)+2 C_{\mathrm{t}}(p) .
$$

3. It is easy to see that

$$
\frac{C_{\mathrm{u}}(n)+C_{\mathrm{su}}(n)}{2}=C_{\tilde{\mathrm{u}}}(n)
$$

where $C_{\tilde{\mathrm{u}}}(n)$ is the number of circulants up to complementarity (and isomorphism), that is, different unordered pairs consisting of an undirected circulant and its complement [13]. Therefore identity (4.1) can be represented in the following simpler form:

$$
C_{\mathrm{sd}}(p)=C_{\tilde{\mathrm{u}}}(p)
$$

It turns into

$$
C_{\mathrm{t}}(p)=C_{\tilde{\mathrm{u}}}(p) \quad \text { if } p \equiv 3 \quad(\bmod 4) .
$$

4.3. We return to identity (3.3). There are subtler analogues of it for undirected and directed circulants. By straightforward observations of numerical data and subsequent numerical verifications with the help of the formulae for prime and twice prime orders (Theorem 1.3, the second, eighth, first and seventh formulae) we arrived at the following somewhat unusual formulae:

4.4 Proposition If $p$ and $q=\frac{p+1}{2}$ are both odd primes, then

$$
\begin{aligned}
4 C_{\mathrm{u}}(p) & =C_{\mathrm{u}}(p+1)+2 \bar{C}_{\mathrm{u}}(2 \tilde{p}+1), \\
2 c_{\mathrm{u}}(p, z) & =\frac{c_{\mathrm{u}}(p+1, z)}{1+z}+\bar{c}_{\mathrm{u}}\left(2 \tilde{p}+1, z^{2^{k}}\right), \\
4 C_{\mathrm{d}}(p) & =C_{\mathrm{d}}(p+1)+2 \bar{C}_{\mathrm{u}}(2 \tilde{p}+1)
\end{aligned}
$$


and

$$
2 c_{\mathrm{d}}(p, z)=\frac{c_{\mathrm{d}}(p+1, z)}{1+z}+\bar{c}_{\mathrm{u}}\left(2 \tilde{p}+1, z^{2^{k}}\right) .
$$

In these equations, $\tilde{p}$ denotes the maximal odd divisor of $p-1$ and

$$
p-1:=2^{k+1} \tilde{p}
$$

Now $\bar{c}_{\mathrm{u}}(2 \tilde{p}+1, z):=c_{\mathrm{u}}(2 \tilde{p}+1, z)$ if $2 \tilde{p}+1$ is a prime, otherwise $\bar{c}_{\mathrm{u}}$ is calculated by the same formula (the second formula in Theorem 1.3) although in this case it does not represent the number of non-isomorphic undirected circulants of order $2 \tilde{p}+1$.

Proof: It is clear that formulae (4.2) and (4.4) follow directly from (4.3) and (4.5) respectively. Formula (4.3) is a direct consequence of (2.1) with $2 m=q-1$ and the corresponding formulae of Theorem 1.3 for orders $p$ and $p+1=2 q$. So in the terminology of Section 2, $\tilde{p}=m^{\prime}$, where $p-1=2(q-1):=4 m$. Formula (4.5) follows similarly but with $m=q-1$.

For instance, by data in Table 2 one can verify that $2 c_{\mathrm{d}}(37, z)=c_{\mathrm{d}}(38, z) /(1+z)+$ $c_{\mathrm{u}}\left(19, z^{2}\right)$. Hence for the valency $r=4$ we have numerically $2(1641+199)=3679+1$, etc.

In particular, by (4.3),

$$
2 C_{\mathrm{u}}(p, 4 r+2)=C_{\mathrm{u}}(p+1,4 r+2)
$$

when $p$ and $\frac{p+1}{2}$ are both odd primes since other terms correspond to undirected circulants of odd orders and odd valency and, thus, vanish.

From (4.2) and (4.4) we obtain the following identity not depending on $\tilde{p}$ :

\subsection{Corollary}

$$
4 C_{\mathrm{d}}(p)-C_{\mathrm{d}}(p+1)=4 C_{\mathrm{u}}(p)-C_{\mathrm{u}}(p+1), \quad p \text { and } \frac{p+1}{2} \text { odd primes }
$$

For example, for $p=13,4 \cdot 352-1400=4 \cdot 14-48=8\left(=2 C_{\mathrm{u}}(7)\right)$. For $p=73$ we obtain rather spectacularly $4 \cdot 65588423374144427520-262353693496577709960=$ $4 \cdot 1908881900-7635527480=120\left(=2 C_{\mathrm{u}}(19)\right)($ moreover, $120=4 \cdot 14602-58288=$ $\left.4 C_{\mathrm{u}}(37)-C_{\mathrm{u}}(38)\right)$.

Identity (4.6) can also be written as

$$
4\left(C_{\mathrm{d}}(p)-C_{\mathrm{u}}(p)\right)=C_{\mathrm{d}}(p+1)-C_{\mathrm{u}}(p+1)
$$




$$
4 C_{\mathrm{d} \backslash \mathrm{u}}(p)=C_{\mathrm{d} \backslash \mathrm{u}}(p+1), \quad p \text { and } \frac{p+1}{2} \text { odd primes, }
$$

where $C_{\mathrm{d} \backslash \mathrm{u}}(n)$ denotes the number of directed circulant graphs that are not undirected graphs.

Similarly from (4.3) and (4.5) we obtain

$$
2(1+z) c_{\mathrm{d} \backslash \mathrm{u}}(p, z)=c_{\mathrm{d} \backslash \mathrm{u}}(p+1, z), \quad p \text { and } \frac{p+1}{2} \text { odd primes, }
$$

or, equivalently,

$$
2\left(C_{\mathrm{d} \backslash \mathrm{u}}(p, r)+C_{\mathrm{d} \backslash \mathrm{u}}(p, r-1)\right)=C_{\mathrm{d} \backslash \mathrm{u}}(p+1, r), \quad p \text { and } \frac{p+1}{2} \text { odd primes. }
$$

Thus, for example, for $p=13$ and $r=5$ we have $C_{\mathrm{d} \backslash \mathrm{u}}(13,5)=66-0=66$, $C_{\mathrm{d} \backslash \mathrm{u}}(13,4)=43-3=40,66+40=106$ and $C_{\mathrm{d} \backslash \mathrm{u}}(14,5)=217-5=2 \cdot 106$.

4.6 Remark Some number theoretic aspects of identities (4.2)-(4.7) together with (3.1)(3.3) are worth considering. There are 21 such pairs of primes $p=2 q-1$ less 1000 . The first six $p$ are 3, 5, 13,37,61 and 73 with their corresponding $q=2,3,7,19,31$ and 37 . These are the sequences M2492 and M0849 in Sloane's Encyclopedia [19] (resp., A005383 and A005382 in its extended on-line version [18]). In number theory, these numbers are called nearly doubled primes, and pairs $(q, p)$ are also known as Cunningham chains of the second kind of length 2 (see, e.g., [7, 15]). By definition, such primes $q$ resemble the familiar Sophie Germain primes, that is, primes $q$ such that $p=2 q+1$ is also prime. The latter primes play a different role in our formulae: the polynomial $\mathcal{J}_{p-1}=\mathcal{J}_{2 q}$ contains the minimal possible (for $p>3$ ) number of terms, four. In Section 7.8 we will discuss some more advanced data concerning nearly doubled primes.

\section{Circulants of prime-squared order}

Throughout this section, $p$ denotes an arbitrary odd prime.

5.1 Theorem $[10,11]$

$$
\begin{aligned}
c_{\mathrm{d}}\left(p^{2}, z\right) & =\left.\mathcal{C}\left(p^{2} ; \mathbf{x}, \mathbf{y}\right)\right|_{\left\{x_{r}:=1+z^{r}, y_{r}:=1+z^{p r}\right\}_{r=1,2, \ldots}} \\
c_{\mathrm{u}}\left(p^{2}, z\right) & =\left.\mathcal{C}^{*}\left(p^{2} ; \mathbf{x}, \mathbf{y}\right)\right|_{\left\{x_{r}:=1+z^{2 r}, y_{r}:=1+z^{2 p r}\right\}_{r=1,2, \ldots}} \\
c_{\mathrm{o}}\left(p^{2}, z\right) & =\left.\mathcal{C}\left(p^{2} ; \mathbf{x}, \mathbf{y}\right)\right|_{\left\{x_{r}:=1, y_{r}:=1\right\}_{r \text { even }},\left\{x_{r}^{2}:=1+2 z^{r}, y_{r}^{2}:=1+2 z^{p r}\right\}_{r} \text { odd }} \\
C_{\mathrm{sd}}\left(p^{2}\right) & =\left.\mathcal{C}\left(p^{2} ; \mathbf{x}, \mathbf{y}\right)\right|_{\left\{x_{r}:=2, y_{r}:=2\right\}_{r \text { even }},\left\{x_{r}:=0, y_{r}:=0\right\}_{r} \text { odd }} \\
C_{\mathrm{su}}\left(p^{2}\right) & =\left.\mathcal{C}^{*}\left(p^{2} ; \mathbf{x}, \mathbf{y}\right)\right|_{\left\{x_{r}:=2, y_{r}:=2\right\}_{r \text { even }},\left\{x_{r}:=0, y_{r}:=0\right\}_{r} \text { odd }} \\
C_{\mathrm{t}}\left(p^{2}\right) & =\left.\mathcal{C}\left(p^{2} ; \mathbf{x}, \mathbf{y}\right)\right|_{\left\{x_{r}:=0, y_{r}:=0\right\}_{r \text { even }},\left\{x_{r}^{2}:=2, y_{r}^{2}:=2\right\}_{r} \text { odd }}
\end{aligned}
$$


where

$$
\mathcal{C}\left(p^{2} ; \mathbf{x}, \mathbf{y}\right):=\frac{1}{p} \mathcal{J}_{p-1}\left(\mathbf{x}^{p+1}\right)-\frac{1}{p} \mathcal{J}_{p-1}(\mathbf{x y})+\mathcal{J}_{p-1}(\mathbf{x}) \mathcal{J}_{p-1}(\mathbf{y})
$$

and

$$
\mathcal{C}^{*}\left(p^{2} ; \mathbf{x}, \mathbf{y}\right):=\frac{1}{p} \mathcal{J}_{\frac{p-1}{2}}\left(\mathbf{x}^{p+1}\right)-\frac{1}{p} \mathcal{J}_{\frac{p-1}{2}}(\mathbf{x y})+\mathcal{J}_{\frac{p-1}{2}}(\mathbf{x}) \mathcal{J}_{\frac{p-1}{2}}(\mathbf{y})
$$

with $\mathbf{x}^{p+1}:=x_{1}^{p+1}, x_{2}^{p+1}, x_{3}^{p+1}, \ldots$ and $\mathbf{x y}:=x_{1} y_{1}, x_{2} y_{2}, x_{3} y_{3}, \ldots$

5.2. Mixed self-complementary circulant graphs. By definition (see Section 3.6),

$$
C_{\mathrm{sd}}^{\text {mixed }}\left(p^{2}\right):=C_{\mathrm{sd}}\left(p^{2}\right)-C_{\mathrm{su}}\left(p^{2}\right)-C_{\mathrm{t}}\left(p^{2}\right) .
$$

According to [11, 14], the number of non-CI (non-Cayley isomorphic) circulants of order $p^{2}$ is

$$
D_{\mathrm{i}}\left(p^{2}\right)=C_{\mathrm{i}}(p)^{2},
$$

where $\mathrm{i} \in\{\mathrm{sd}, \mathrm{su}, \mathrm{t}\}$. We recall that a circulant is said to be non-CI if there exists a circulant isomorphic but not Cayley isomorphic to it. A Cayley isomorphism means an isomorphism that is induced by an automorphism of the underlying group $\mathbb{Z}_{n}$.

\subsection{Proposition}

$$
C_{\mathrm{sd}}^{\text {mixed }}\left(p^{2}\right)=2 C_{\mathrm{su}}(p) C_{\mathrm{t}}(p)
$$

and

$$
C_{\mathrm{sd}}^{\text {mixed }}\left(p^{2}\right)=D_{\mathrm{sd}}\left(p^{2}\right)-D_{\mathrm{su}}\left(p^{2}\right)-D_{\mathrm{t}}\left(p^{2}\right),
$$

that is, the mixed self-complementary circulants of order $p^{2}$ are exactly the non-CI mixed self-complementary circulants.

Proof: We make use of an algebraic property of self-complementary circulants of primepower order. According to a result announced by Li [12] (Theorem 3.3), if $\Gamma$ is a selfcomplementary circulant of order $p^{2}$ then one of the following holds.

- $\Gamma$ can be obtained by means of the well-known (alternating cycle) construction discovered by Sachs and Ringel.

- $\Gamma=\Gamma_{1}\left[\Gamma_{2}\right]$ where $\Gamma_{1}$ and $\Gamma_{1}$ are self-complementary circulants of order $p$. Here $\Gamma_{1}\left[\Gamma_{2}\right]$ is the composition (called also the wreath or lexicographic product) defined as follows: 
in $\Gamma_{1}$ we replace each vertex by a copy of $\Gamma_{2}$; each edge of $\Gamma_{1}$ gives rise to the edges connecting all pairs of vertices from the two corresponding copies of $\Gamma_{2}$.

The first construction generates only undirected circulants or tournaments (cf. [14]); moreover, all of them are CI. Now, there is no mixed self-complementary circulant of order $p$ (this is identity (3.7)). Therefore the second construction gives rise to a mixed graph if and only if one of the factors is an undirected self-complementary circulant and the other factor is a tournament. This proves (5.3). Further, all self-complementary circulants $\Gamma=\Gamma_{1}\left[\Gamma_{2}\right]$ are non-CI [14]. This, together with (5.2), proves (5.4) (moreover, this proves (5.2) since the composition of two undirected circulants is undirected and the composition of two tournaments is a tournament).

It would be interesting to find an analytical derivation of these equations with the help of Theorem 5.1.

By (5.1) we have

\subsection{Corollary}

$$
C_{\mathrm{sd}}\left(p^{2}\right)-C_{\mathrm{su}}\left(p^{2}\right)-C_{\mathrm{t}}\left(p^{2}\right)=C_{\mathrm{sd}}(p)^{2}-C_{\mathrm{su}}(p)^{2}-C_{\mathrm{t}}(p)^{2} .
$$

5.5 Example $p=13$. By Theorem 5.1, $C_{\mathrm{sd}}\left(13^{2}\right)=123992391755402970674764$, $C_{\text {su }}\left(13^{2}\right)=56385212104$ and $C_{\mathrm{t}}\left(13^{2}\right)=123992391755346585462636$. It follows that $C_{\mathrm{sd}}^{\text {mixed }}\left(13^{2}\right)=24$. Now $C_{\mathrm{sd}}(13)^{2}=8^{2}=64, C_{\mathrm{su}}(13)^{2}=2^{2}=4, C_{\mathrm{t}}(13)^{2}=6^{2}=36$ and $64-4-36=24=2 \cdot 2 \cdot 6$.

By (3.7) (or, instead, by (5.1) and (5.3)), identity (5.5) can be represented as follows:

$$
C_{\mathrm{sd}}\left(p^{2}\right)=C_{\mathrm{su}}\left(p^{2}\right)+C_{\mathrm{t}}\left(p^{2}\right)+2 C_{\mathrm{su}}(p) C_{\mathrm{t}}(p) .
$$

We note also that if $p \equiv 3(\bmod 4)$, then $C_{\mathrm{su}}(p)$ and $C_{\mathrm{su}}\left(p^{2}\right)$ vanish by (3.4), and identity (5.6) turns into (3.5) for $n=p^{2}$.

\section{Alternating sums}

Alternating sums serve as one further source of formal identities. First consider directed circulants of prime order. Take the generating function $c_{\mathrm{d}}(p, t)$ and put $t:=-1$. By Theorem 1.3 we see that the result is equal to $C_{\mathrm{sd}}(p)$. By Theorem 5.1, the same equality is valid for the orders $n=p^{2}$. Moreover, by formulae given in [11] it is valid for arbitrary odd square-free orders. Thus, for prime-squared and square-free $n$ we have

$$
c_{\mathrm{d}}(n,-1)=C_{\mathrm{sd}}(n)
$$


The corresponding result holds for the same $n$ for undirected circulants with respect to the substitution $t^{2}:=-1$, or $t:=\sqrt{-1}$ :

$$
\left.c_{\mathrm{u}}(n, t)\right|_{t^{2}:=-1}=C_{\mathrm{su}}(n) .
$$

It is natural to suggest that both formulae are valid in general:

6.1. Conjecture. Identities (6.1) and (6.2) hold for any odd order $n$.

Trivially (by complementation), identity (6.1) holds also for even $n$, and (6.2) holds for $n \equiv 3(\bmod 4)$, see (3.9) and (3.10). Identity (6.2) is also valid for $n=45$ as numerical data [16] show.

The behaviour of oriented circulant graphs is different. Numerical observations show that

$$
c_{\mathrm{o}}(n,-1)=0
$$

if $n$ has at least one prime divisor $p \equiv 3(\bmod 4)$, otherwise

$$
c_{\mathrm{o}}(n,-1)=1
$$

These identities hold for prime $n=p$ by Theorem 1.3, for odd square-free $n$ by [11] and for $n=p^{2}$ by Theorem 5.1. Again we conjecture them to be valid for all odd $n$.

For even square-free $n$ we found that identity (6.3b) holds if $n=2 n^{\prime}, n^{\prime}$ odd, and (6.3a) holds if $n=4 n^{\prime}, n^{\prime}$ square-free. The behaviour of $c_{0}(n,-1)$ for $n=8 n^{\prime}, n^{\prime}>1$, remains unknown.

Identities (6.1) and (6.2) for prime $n=p$ transform (4.1) into the following equality:

$$
2 c_{\mathrm{d}}(p,-1)=c_{\mathrm{u}}(p, 1)+c_{\mathrm{u}}(p, \sqrt{-1}) .
$$

6.2. Even- and odd-valent circulants. Due to (6.1) and (6.2) we can find simple expressions for the numbers of circulants of (non-specified) even (and, resp., odd) valency; for undirected circulants we consider only odd orders and mean even and odd semi-valencies, that is, valencies congruent, respectively, to 0 and 2 modulo 4 . We use the superscript e and o to denote these numbers. Now, formula (6.1) is nothing than $C_{\mathrm{d}}^{\mathrm{e}}(n)-C_{\mathrm{d}}^{\mathrm{o}}(n)=C_{\mathrm{sd}}(n)$. Since $C_{\mathrm{d}}^{\mathrm{e}}(n)+C_{\mathrm{d}}^{\mathrm{o}}(n)=C_{\mathrm{d}}(n)$, we obtain

$$
C_{\mathrm{d}}^{\mathrm{e}}(n)=\frac{C_{\mathrm{d}}(n)+C_{\mathrm{sd}}(n)}{2}
$$

and

$$
C_{\mathrm{d}}^{\mathrm{o}}(n)=\frac{C_{\mathrm{d}}(n)-C_{\mathrm{sd}}(n)}{2} .
$$

So, these expressions hold for square-free, prime-squared and even $n$ and are assumed to hold for all orders. 
Similarly, (6.2) gives rise to

$$
C_{\mathrm{u}}^{\mathrm{e}}(n)=\frac{C_{\mathrm{u}}(n)+C_{\mathrm{su}}(n)}{2},
$$

and

$$
C_{\mathrm{u}}^{\mathrm{o}}(n)=\frac{C_{\mathrm{u}}(n)-C_{\mathrm{su}}(n)}{2}
$$

for undirected circulants of odd orders and even and, resp., odd semi-valency. Equations (6.6e) and (6.6o) remain unproven unless $n$ is square-free or prime-squared or congruent to 3 modulo 4 .

Clearly the respective expressions can be extracted from (6.3a) and (6.3b) for oriented circulants.

Comparing formula (6.6e) for prime $n=p$ with (4.1) we obtain the following curious identity:

$$
C_{\mathrm{u}}^{\mathrm{e}}(p)=C_{\mathrm{sd}}(p)
$$

This equation directly generalizes identity (4.1a) to $p \equiv 1(\bmod 4)$ because $C_{\mathrm{u}}^{\mathrm{e}}(p)=$ $C_{\mathrm{u}}(p) / 2$ for $p \equiv 3(\bmod 4)$. By $(6.1)$, this may also be written as $C_{\mathrm{u}}^{\mathrm{e}}(p)=c_{\mathrm{d}}(p,-1)$. Finally, identities (4.1c) and (6.7) imply

$$
C_{\mathrm{u}}^{\mathrm{e}}(p)=C_{\tilde{\mathrm{u}}}(p) .
$$

\section{Discussion}

As we saw above, the enumerative theory of circulants is full of hidden inter-dependencies. Table 3 in the Appendix contains a summary of previous and new identities.

We expect that there should exist further generalizations of the obtained identities for other classes of circulant graphs, first of all, for multigraphs and graphs with coloured or marked edges.

7.1. Let $C_{\mathrm{sd}}^{\prime}(n)$ denote the number of directed self-complementary $n$-circulants whose automorphism group coincides with $Z(n)$. Such circulants are called strong. The numbers of strong circulants can be counted for prime $n=p$ by the following simple formula [4] (cf. also [3]):

$$
C_{\mathrm{sd}}^{\prime}(p)=\frac{1}{p-1} \sum_{d \mid \frac{p-1}{2}, d \text { odd }} \mu(d) 2^{(p-1) / 2 d}
$$

where $\mu(d)$ is the number theoretic Möbius function. A similar formula is valid for undirected self-complementary circulants without additional automorphisms [14]. Every strong 
self-complementary circulant digraph is a tournaments, and as calculations show, the values of $C_{\mathrm{sd}}^{\prime}(p)$ are close to those of $C_{\mathrm{t}}(p)$. Are there any interesting identities containing $C_{\mathrm{sd}}^{\prime}(p)$ ?

7.2. Is it possible to give a bijective proof of identity (4.1) or one of its clones (including $(6.7)$ and $\left.\left(6.7^{\prime}\right)\right)$ ? This question looks especially intriguing in view of the fact that general circulant graphs, unlike self-complementary circulants, are naturally partitioned by valency. Hence such a bijection would introduce a certain external graduation ("pseudo-valency") into the class of self-complementary circulant digraphs of prime order. Self-complementary circulants possess their own natural graduations. Such is, for instance, the one defined by the number of orbits of the automorphism group in its action on the set of arcs. Is there a natural graduation that corresponds to the valency of undirected circulants? We could put formally $x_{r}:=1+z^{r}, r=1,2, \ldots$, in (2.4) instead of $x_{r}:=2$. But is there a natural combinatorial interpretation of the coefficients of the left hand-side polynomial thus obtained?

7.3. In general, analytical identities are characteristic for enumerators of selfcomplementary graphs of diverse classes. Such results can be found in numerous publications. We refer to surveys by Robinson [17] and Farrugia [6]. In the latter, several open questions are also posed. In particular, the problem $\mathrm{K}$ in Sect. 7.64 is just the problem of finding a natural bijection for identity (3.2).

7.4. Open question for mixed circulants. Is identity (3.5) valid for the orders all whose prime divisors are congruent to 3 modulo 4? In other words (in view of (3.4)), are there mixed self-complementary circulants of such orders? We conjecture that mixed selfcomplementary circulants of order $n$ exist if and only if $n$ is odd composite and has a prime divisor $p \equiv 1(\bmod 4)$. If so, then, moreover, identity (3.7) holds if and only if all prime divisors of $n$ are congruent to 3 modulo 4 .

This conjecture is valid for square-free orders, and it can also be proved for the primepower orders $n=p^{k}$.

7.5. Here are the four non-isomorphic mixed self-complementary circulants of order 15 mentioned in Section 3.6:

$$
\begin{aligned}
& X\left(\Gamma_{1}\right)=\{1,-2,4,-8,3,-3,5\}, \quad X\left(\Gamma_{3}\right)=\{1,-1,4,-4,6,-6,5\}, \\
& X\left(\Gamma_{2}\right)=\{1,-2,4,-8,3,-3,-5\}, \quad X\left(\Gamma_{4}\right)=\{1,-1,4,-4,6,-6,-5\} .
\end{aligned}
$$

It is easy to see that $\Gamma_{2} \cong \vec{z}_{3}\left[z_{5}\right]$ and $\Gamma_{3} \cong z_{5}\left[\overrightarrow{z_{3}}\right]$, where [ ] denotes the compositions (see 5.3), $\vec{z}_{3}$ a directed triangle and $z_{5}$ an undirected 5-cycle.

The next suitable order is 25 ; there are only $2=214-205-7$ mixed self-complementary circulants.

7.6. Conjectural (for arbitrary $n$ ) identity (3.8) can be interpreted as follows. Let $\Gamma$ be an arbitrary undirected circulant graph of order $2 n$ and even valency. This means that its connection set $X$ does not contain $n$. Let $\Gamma^{\prime}$ be another circulant graph isomorphic to $\Gamma$. 
Then the corresponding odd-valent circulant graphs with the connection sets $X(\Gamma) \cup\{n\}$ and $X\left(\Gamma^{\prime}\right) \cup\{n\}$ are, presumably, also isomorphic. We assume that the following stronger assertion is valid:

Conjecture. If $\Gamma$ and $\Gamma^{\prime}$ are two isomorphic undirected circulant graphs of order $2 n$, then there exists an isomorphism between them which is also an automorphism of the 1-valent circulant graph $\Gamma_{0}$ with the connection set $X\left(\Gamma_{0}\right)=\{n\}$.

The graph $\Gamma_{0}$ is a perfect matching (the set of "spokes" in $\mathbb{Z}_{2 n}$ ). The point is that an undirected circulant of odd valency can contain a lot of perfect matchings, and a particular isomorphism of it does not have to preserve this specific matching. Moreover, the assertion of the conjecture is not valid for Cayley graphs in general.

The conjecture is evident for CI circulant graphs and it is in conformity with the wellknown smallest example of a non-CI circulant graph constructed by Elspas and Turner [5]. Namely, the undirected circulant graphs of order 16 and valency 6 with the connection sets $X=\{1,2,7,9,14,15\}$ and $X^{\prime}=\{2,3,5,11,13,14\}$ are isomorphic but not Cayley isomorphic. The isomorphism

$$
\begin{cases}i \mapsto i & \text { for even } i \\ i \mapsto i+4 & \text { for odd } i\end{cases}
$$

between them [5] clearly preserves the set of spokes $\Gamma_{0}$, where $X\left(\Gamma_{0}\right)=\{8\}$.

7.7. Can identities (4.2)-(4.7) (as well as (3.1)-(3.3)) be treated bijectively? What is then the meaning of the sum or the corresponding difference? This question is particularly curious for (4.2) and (4.4) in the case of small $\tilde{p}$. The existence of such a treatment seems doubtful at least for composite $2 \tilde{p}+1$. In this respect, identities (4.6) and (4.7) appear to be more promising.

7.8. Number theoretic digression. Return to 4.6. It is commonly believed that the set of nearly doubled primes is infinite. Moreover, there is a conjecture that the number $\pi_{\text {ndp }}(N)$ of such primes $p<N$ grows asymptotically with $N$ as $\frac{C N}{(\log N)^{2}}$ where $C=1.320 \ldots$ is twice the familiar twin prime constant (curiously, for $N=10^{s}$, this fraction is close to $\frac{10^{s}}{4 s^{2}}$ ). Recall that the number $\pi(N)$ of all primes $p<N$ grows approximately as $\frac{N}{\log N-1}$.

At present, a lot of efforts in computational number theory are devoted to the search for Cunningham chains of huge numbers, especially long chains (see, e.g., [7]). In particular, the familiar program proth.exe by Y. Gallot allows to effectively verify the primality of numbers $m \cdot 2^{k}+1$ with a fixed $m$. Keeping in mind identities (4.2)-(4.5) we are especially interested in nearly doubled primes $q=m \cdot 2^{k}+1$ and $p=m \cdot 2^{k+1}+1$ with small $m:=\tilde{p}$. In general it is easy to see that such a pair $q, p$ can exist only if $3 \mid m$. Here are the current numerical results for $m \leq 27$.

Pairs of primes $q, p$ of the form $3 \cdot 2^{k}+1$ occur twice for $k \leq 2000000$ : only with $k=1,2$ and $k=5,6(p=193)$; see the sequence M1318 in [19] (or A002253 [18]). 
Pairs of primes $q, p$ of the form $9 \cdot 2^{k}+1$ occur four times for $k \leq 350000$ : with $k=1,2, k=2,3, k=6,7$ and $k=42,43$; see M0751 (A002256).

Pairs of primes $q, p$ of the form $15 \cdot 2^{k}+1$ occur three times for $k \leq 270000$ : with $k=1,2, k=9,10$ and $k=37,38$; see M1165 (A002258).

Pairs of primes $q, p$ of the form $21 \cdot 2^{k}+1$ occur three times for $k \leq 262000$ : with $k=4,5, k=16,17$ and $k=128,129$ (see A032360 [18]).

Pairs of primes $q, p$ of the form $27 \cdot 2^{k}+1$ occur twice for $k \leq 265000$ : with $k=19,20$ and $k=46,47$ (see A032363 [18]). This case gives rise to the least possible composite value of $2 \tilde{p}+1,55$. So, for the first time it arises for $p=2 q-1=27 \cdot 2^{20}+1=28311553$.

Clearly $2 \tilde{p}+1=q$ if 8 does not divide $p-1$. For $p<2000,2 \tilde{p}+1$ turns out to be composite only in three cases. $q=229, p=457$ is the least case; here $\tilde{p}=57$ and $2 \tilde{p}+1=115$.

By numerical data we found out that no Cunningham chain exists for $m=51,87$ and 93 at least for $k<170000$. Actually there are multipliers $m=\tilde{p}$, called the Sierpinski numbers, such that all $n=m \cdot 2^{k}+1, k=1,2, \ldots$, are composite, and Sierpinski numbers may be divisible by 3 . But are there other odd $m$ divisible by 3 such that no Cunningham chain exists for them? The answer to this question is affirmative, and $m=66741$ is the least known value (found by Y. Gallot, private communication; the point is that if $k$ is an even number, then $66741 \cdot 2^{k}+1$ is divisible by $5,7,13,17$ or 241$) .{ }^{1}$

Here are two remarkable nearly doubled primes: ${ }^{2}$

$141 \cdot 2^{k}+1$ are prime for $k=555,556$;

$975 \cdot 2^{k}+1$ are prime for $k=6406,6407$.

7.9. For alternating sums, identities (6.1), (6.2), (6.5) and (6.6) are rather typical; cf., e.g., the paper [13], where other examples of even- and odd-specified quantities and the corresponding half-sum expressions for them are given.

7.10. Finally, instead of equalities, we touch one important type of inequalities which are frequently proved analytically. I conjecture that the sequence of the numbers $C_{\mathrm{u}}(p, 2 r)$, $1<r<(p-1) / 2$, is logarithmically concave, that is

$$
C_{\mathrm{u}}(n, 2 r)^{2} \geq C_{\mathrm{u}}(n, 2 r-2) C_{\mathrm{u}}(n, 2 r+2)
$$

for any prime order $n=p$ and $1<r<(n-1) / 2$. In other words, the sequence of ratios $C_{\mathrm{u}}(p, 2 r) / C_{\mathrm{u}}(p, 2 r+2)$ is increasing except for the first and the last member. For composite orders this does not necessarily hold. In particular, the opposite inequality holds for $r=2$ when $n=27,121$ and 169. However I do not know counterexamples for square-free orders.

\section{Appendix: Numerical results and summary}

Tables 1 and 2 contain relevant numerical data obtained by Theorems 1.3 and 5.1 (they partially reproduce data from [11]). 
Table 1. Non-isomorphic circulant graphs.

\begin{tabular}{|c|c|c|c|c|c|c|}
\hline$n$ & $C_{\mathrm{d}}(n)$ & $C_{\mathrm{u}}(n)$ & $C_{\mathrm{o}}(n)$ & $C_{\mathrm{sd}}(n)$ & $C_{\mathrm{su}}(n)$ & $C_{\mathrm{t}}(n)$ \\
\hline 2 & 2 & 2 & 1 & 0 & 0 & 0 \\
\hline 3 & 3 & 2 & 2 & 1 & 0 & 1 \\
\hline 4 & 6 & 4 & 2 & 0 & 0 & 0 \\
\hline 5 & 6 & 3 & 3 & 2 & 1 & 1 \\
\hline 6 & 20 & 8 & 5 & 0 & 0 & 0 \\
\hline 7 & 14 & 4 & 6 & 2 & 0 & 2 \\
\hline 8 & 46 & 12 & 7 & 0 & 0 & 0 \\
\hline 9 & 51 & 8 & 16 & 3 & 0 & 3 \\
\hline 10 & 140 & 20 & 21 & 0 & 0 & 0 \\
\hline 11 & 108 & 8 & 26 & 4 & 0 & 4 \\
\hline 12 & 624 & 48 & 64 & 0 & 0 & 0 \\
\hline 13 & 352 & 14 & 63 & 8 & 2 & 6 \\
\hline 14 & 1400 & 48 & 125 & 0 & 0 & 0 \\
\hline 15 & 2172 & 44 & 276 & 20 & 0 & 16 \\
\hline 17 & 4116 & 36 & 411 & 20 & 4 & 16 \\
\hline 18 & 22040 & 192 & 1105 & 0 & 0 & 0 \\
\hline 19 & 14602 & 60 & 1098 & 30 & 0 & 30 \\
\hline 20 & 68016 & 336 & 2472 & 0 & 0 & 0 \\
\hline 21 & 88376 & 200 & 4938 & 88 & 0 & 88 \\
\hline 22 & 209936 & 416 & 5909 & 0 & 0 & 0 \\
\hline 23 & 190746 & 188 & 8054 & 94 & 0 & 94 \\
\hline 25 & 839094 & 423 & 26577 & 214 & 7 & 205 \\
\hline 26 & 2797000 & 1400 & 44301 & 0 & 0 & 0 \\
\hline 28 & 11276704 & 3104 & 132964 & 0 & 0 & 0 \\
\hline 29 & 9587580 & 1182 & 170823 & 596 & 10 & 586 \\
\hline 30 & 67195520 & 8768 & 597885 & 0 & 0 & 0 \\
\hline 31 & 35792568 & 2192 & 478318 & 1096 & 0 & 1096 \\
\hline 33 & 214863120 & 6768 & 2152366 & 3280 & 0 & 3280 \\
\hline 34 & 536879180 & 16460 & 2690421 & 0 & 0 & 0 \\
\hline 35 & 715901096 & 11144 & 5381028 & 5560 & 0 & 5472 \\
\hline 37 & 1908881900 & 14602 & 10761723 & 7316 & 30 & 7286 \\
\hline 38 & 7635527480 & 58288 & 21523445 & 0 & 0 & 0 \\
\hline 39 & 11454711464 & 44424 & 48427776 & 21944 & 0 & 21856 \\
\hline 41 & 27487816992 & 52488 & 87169619 & 26272 & 56 & 26216 \\
\hline 42 & 183264019200 & 355200 & 290566525 & 0 & 0 & 0 \\
\hline 43 & 104715443852 & 99880 & 249056138 & 49940 & 0 & 49940 \\
\hline 44 & 440020029120 & 432576 & 523020664 & 0 & 0 & 0 \\
\hline 46 & 1599290021720 & 762608 & 1426411805 & 0 & 0 & 0 \\
\hline 47 & 1529755490574 & 364724 & 2046590846 & 182362 & 0 & 182362 \\
\hline 49 & 6701785562464 & 798952 & 6724513104 & 399472 & 0 & 399472 \\
\hline 50 & 28147499352824 & 3356408 & 14121476937 & 0 & 0 & 0 \\
\hline
\end{tabular}


Table 2. Enumeration of circulant graphs by valency (for selective orders).

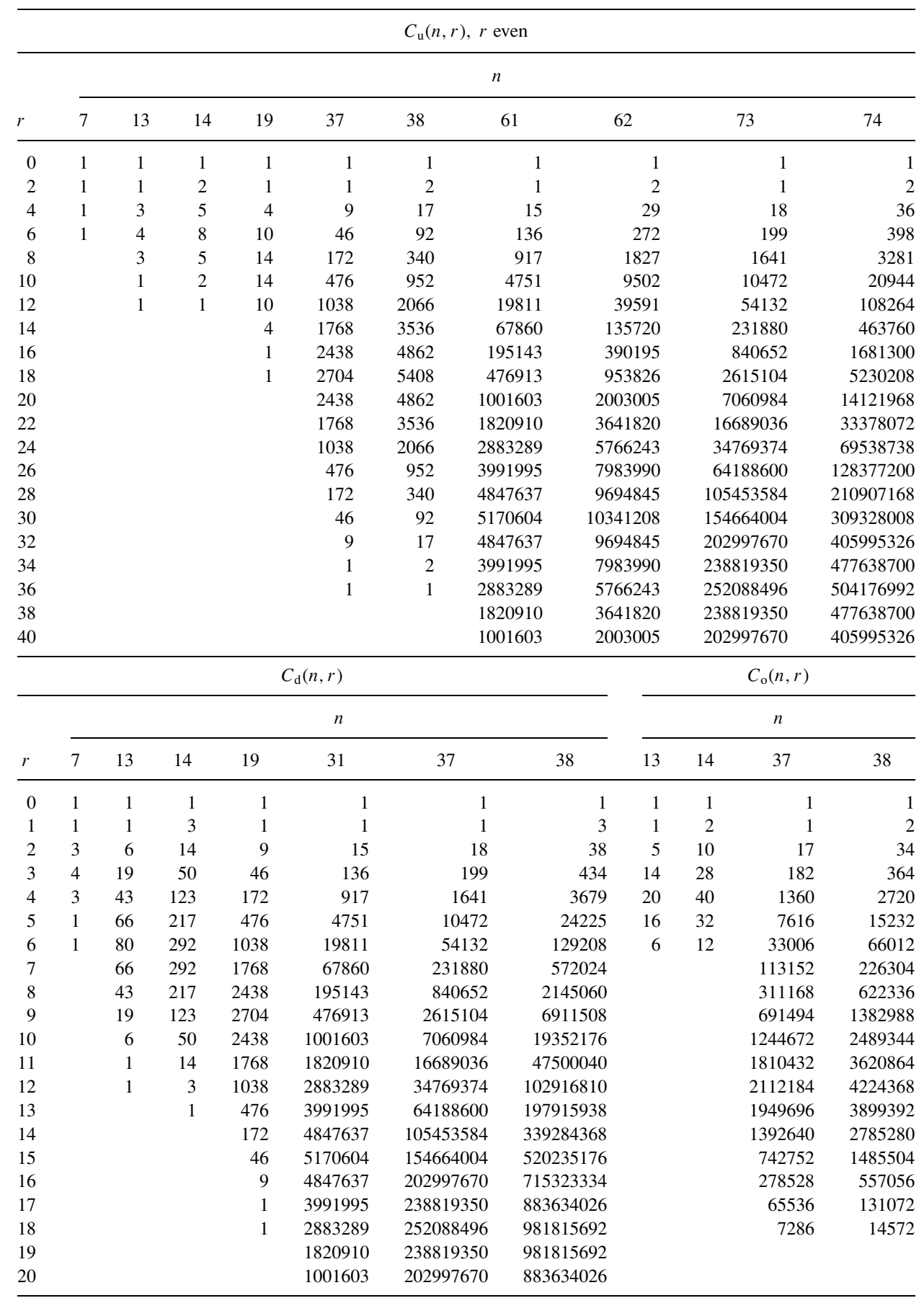


Table 3. Systematized list of identities.

\begin{tabular}{|c|c|c|c|c|c|c|}
\hline No. & Formula & Orders $^{\mathrm{a}}$ & Restrictions & Types & Proof & Ref. \\
\hline \multicolumn{7}{|c|}{ For self-complementary circulants: } \\
\hline 1 & (3.4) & $n$ & $\exists p \mid n, p \equiv 3(\bmod 4)$ & su & Combin. & [8] \\
\hline & & & & & Algebraic & [1] \\
\hline & & $p$ or $p^{2}$ & $p \equiv 3(\bmod 4)$ & & Analytical & {$[10]$} \\
\hline 2 & $(3.5)$ & $p$ or $p^{2}$ & $p \equiv 3(\bmod 4)^{\mathrm{b}}$ & $\mathrm{t}, \mathrm{sd}$ & $\begin{array}{l}\text { Analytical } \\
\Leftarrow(3.4),(5.3)\end{array}$ & $\begin{array}{c}{[10]} \\
(\mathrm{New})\end{array}$ \\
\hline \multirow[t]{2}{*}{3} & $(3.7)$ & $p$ & - & su, t, sd & Algebraic & [4] \\
\hline & & $n$ & $n=p, \ldots{ }^{\mathrm{b}}$ & & Analytical & New \\
\hline 4 & $(4.1)$ & $p$ & - & $\mathrm{u}, \mathrm{su}, \mathrm{sd}$ & Analytical & New \\
\hline 5 & (4.1a) & $p$ & $p \equiv 3(\bmod 4)$ & $\mathrm{u}, \mathrm{sd}$ & $\Leftarrow(4.1),(3.4)$ & (New) \\
\hline 6 & $(4.1 b)$ & $p$ & - & $\mathrm{u}, \mathrm{su}, \mathrm{t}$ & $\Leftarrow(4.1),(3.7)$ & (New) \\
\hline 7 & $(4.1 c)$ & $p$ & - & $\tilde{\mathrm{u}}, \mathrm{sd}$ & $\Leftarrow(4.1)$ & (New) \\
\hline 8 & $(4.1 d)$ & $p$ & $p \equiv 3(\bmod 4)$ & $\tilde{u}, t$ & $\Leftarrow(4.1 \mathrm{c})$ & (New) \\
\hline 9 & $(5.5)$ & $p, p^{2}$ & - & su, t, sd & $\Leftarrow(5.2),(5.4)$ & (New) \\
\hline 10 & (5.6) & $p, p^{2}$ & - & $\mathrm{su}, \mathrm{t}, \mathrm{sd}$ & $\Leftarrow(3.7),(5.5)$ & (New) \\
\hline 11 & (3.6) & $p, q$ & $p+1=2 q \equiv 6(\bmod 8)$ & su, t & $\Leftarrow(3.2),(3.7)$ & (New) \\
\hline 12 & $(3.2)$ & $p, q$ & $p+1=2 q$ & su, sd & Analytical & {$[10]$} \\
\hline \multicolumn{7}{|c|}{ Other valency independent: } \\
\hline 13 & $\left(3.1^{\prime}\right)$ & $p, q$ & $p+1=2 q$ & $\mathrm{u}, \mathrm{d}$ & $\Leftarrow(3.1)$ & {$[10]$} \\
\hline 14 & $\left(3.3^{\prime}\right)$ & $p, p+1$ & $p+1=2 q$ & o & $\Leftarrow(3.3)$ & (New) \\
\hline 15 & $(4.2)$ & $p, p+1$ & $p+1=2 q$ & $\mathrm{u}$ & $\Leftarrow(4.3)$ & (New) \\
\hline 16 & $(4.4)$ & $p, p+1$ & $p+1=2 q$ & $\mathrm{u}, \mathrm{d}$ & $\Leftarrow(4.5)$ & (New) \\
\hline 17 & (4.6) & $p, p+1$ & $p+1=2 q$ & $\mathrm{u}, \mathrm{d}$ & $\Leftarrow(4.2),(4.4)$ & (New) \\
\hline 18 & $\left(4.6^{\prime}\right)$ & $p, p+1$ & $p+1=2 q$ & $d \backslash u$ & $\Leftarrow(4.6)$ & (New) \\
\hline \multicolumn{7}{|c|}{ By valency: } \\
\hline 19 & (3.1) & $p, q$ & $p+1=2 q$ & $\mathrm{u}, \mathrm{d}$ & Analytical & {$[10]$} \\
\hline 20 & (3.3) & $p, p+1$ & $p+1=2 q$ & o & Analytical & New \\
\hline 21 & (4.3) & $p, p+1$ & $p+1=2 q$ & $\mathrm{u}$ & Analytical & New \\
\hline 22 & $\left(4.3^{\prime}\right)$ & $p, p+1$ & $p+1=2 q$ & $\mathrm{u}$ & $\Leftarrow(4.3)$ & (New) \\
\hline 23 & $(4.5)$ & $p, p+1$ & $p+1=2 q$ & $\mathrm{u}, \mathrm{d}$ & Analytical & New \\
\hline 24 & (4.7) & $p, p+1$ & $p+1=2 q$ & $d \backslash u$ & $\Leftarrow(4.3),(4.5)$ & (New) \\
\hline \multirow[t]{2}{*}{25} & (3.8) & $2 n$ & $n<27$ & $\mathrm{u}$ & Exh. search & [16] \\
\hline & & & square-free ${ }^{c}$ & & Analytical & [11] \\
\hline 26 & $(3.9)$ & $2 n+1$ & - & $\mathrm{u}$ & Trivial & - \\
\hline 27 & $(3.10)$ & $n$ & - & $\mathrm{u} ; \mathrm{d}$ & Trivial & - \\
\hline \multicolumn{7}{|c|}{ Alternating: } \\
\hline 28 & (6.1) & $n$ & $p^{2}$ or sq. free ${ }^{\mathrm{d}}$ & $d, s d$ & Analytical & New \\
\hline 29 & $(6.2)$ & $n$ & $p^{2}$ or sq. free ${ }^{\mathrm{d}}$ & $\mathrm{u}, \mathrm{su}$ & Analytical & New \\
\hline 30 & (6.3) & $n$ & $p^{2}$ or sq. free $\mathrm{d}^{\mathrm{d}, \mathrm{e}}$ & o & Analytical & New \\
\hline 31 & (6.4) & $p$ & - & $\mathrm{u}, \mathrm{d}$ & $\Leftarrow(6.1),(4.1)$ & (New) \\
\hline \multicolumn{7}{|c|}{ Miscellaneous (non-CI, mixed, of even semi-valency, ....): } \\
\hline 32 & $(5.2)$ & $p, p^{2}$ & - & $\mathrm{su} ; \mathrm{t} ; \mathrm{sd}$ & Algebraic & [14] \\
\hline 33 & (5.3) & $p, p^{2}$ & - & su, t, sd & Algebraic & New \\
\hline 34 & (5.4) & $p^{2}$ & - & $\mathrm{su}, \mathrm{t}, \mathrm{sd}$ & Algebraic & New \\
\hline 35 & (6.7) & $p$ & - & $\mathrm{u}^{\mathrm{e}}, \mathrm{sd}$ & $\Leftarrow(6.1),(4.1)$ & (New) \\
\hline 36 & $\left(6.7^{\prime}\right)$ & $p$ & - & $\mathrm{u}^{\mathrm{e}}, \tilde{u}$ & $\Leftarrow(6.7),(4.1 \mathrm{c})$ & (New) \\
\hline
\end{tabular}

${ }^{\mathrm{a}} p$ and $q$ are odd primes.

${ }^{\mathrm{b}}$ Holds also for $n=p^{k}$ and square-free $n$ with all prime divisors $p \equiv 3(\bmod 4)$.

Is conjectured to hold for arbitrary $n$ with all such prime divisors.

${ }^{\mathrm{c}}$ Is conjectured to hold for arbitrary even orders and odd valencies.

${ }^{\mathrm{d}}$ Is conjectured to hold for arbitrary odd orders.

${ }^{\mathrm{e}}$ There is a corresponding conjecture for arbitrary even orders $n, 8+n$. 


\section{Acknowledgment}

I am thankful to the referees for reading my text attentively and suggesting many helpful corrections.

\section{Notes}

1. Other details can be found at the site www . primepuzzles .net/problems/prob_036.htm (Problem 36. The Liskovets—Gallot numbers) maintained by C. Rivera.

2. They, together with the latest bounds for $k$ given above, are taken from the corresponding lists maintained in the WWW by W. Keller and N. S. A. Melo, see www . prothsearch. net/riesel.html; cf. also [2].

\section{References}

1. B. Alspach, J. Morris, and V. Vilfred, "Self-complementary circulant graphs," Ars Combinatoria 53 (1999), 187-191.

2. R. Baillie, "New primes of the form $k \cdot 2^{n}+1$," Math. Comput. 33(148) (1979), 1333-1336.

3. C.Y. Chao and J.G. Wells, "A class of vertex-transitive digraphs," J. Combin. Th. B 32(3) (1982), 336-346.

4. G.L. Chia and C.K. Lim, "A class of self-complementary vertex-transitive digraphs," J. Graph Th. 10(2) (1986), 241-249.

5. B. Elspas and J. Turner, "Graphs with circulant adjacency matrices," J. Combin. Th. 9(3) (1970), 297-307.

6. A. Farrugia, Self-Complementary Graphs and Generalisations: A Comprehensive Reference Manual, Master's Thesis, Univ. of Malta (1999) (currently available at http://www.math.uwaterloo. $\mathrm{ca} /{ }^{\sim}$ afarrugia/sc-graph.html).

7. T. Forbes, "Prime clasters and Cunningham chains," Math. Comput. 68(228) (1999), 1739-1747.

8. D. Fronček, A. Rosa and J. Širáň, “The existence of selfcomplementary circulant graphs,” Europ. J. Combin. 17(7) (1996), 625-628.

9. F. Harary, Graph Theory, Addison-Wesley, Reading, MA, 1969.

10. M. Klin, V. Liskovets, and R. Pöschel, "Analytical enumeration of circulant graphs with primesquared number of vertices," Sém. Lotharing. Combin., 36 (1996), B36d (currently available at http://www.mat.univie.ac.at/ slc/).

11. M. Klin, V. Liskovets and R. Pöschel, "On the analytical enumeration of circulant graphs," Technical Report MATH-AL-07-2003, Tu-Dresden, July 2003.

12. C.H. Li, "On finite graphs that are self-complementary and vertex-transitive," Australas. J. Combin. 18 (1998), $147-155$.

13. V.A. Liskovets, "Some easily derivable integer sequences," J. Integer Seq. 3 (2000), Article 00.2.2 (currently available at http://www . math. uwaterloo.ca/JIS/).

14. V. Liskovets and R. Pöschel, "Non-Cayley-isomorphic self-complementary circulant graphs," J. Graph Th. 34(2) (2000), 128-141.

15. G. Löh, "Long chains of nearly doubled primes," Math. Comput. 53(188) (1989), 751-759.

16. B.D. McKay, Personal communication (1995).

17. R.W. Robinson, "Counting graphs with a duality property," Combinatorics, Proc. 8th Brit. Comb. Conf., Swansea 1981, Lond. Math. Soc. Lect. Notes Ser. 52 (1981), 156-186.

18. N.J.A. Sloane, The On-Line Encyclopedia of Integer Sequences, published electronically at http://www.research.att.com/ ${ }^{\sim}$ jjas/sequences/

19. N.J.A. Sloane and S. Plouffe, The Encyclopedia of Integer Sequences, Academic Press, San Diego, 1995. 